\title{
ADVECTION OF PASSIVE TRACERS IN THE ATMOSPHERE: BATCHELOR SCALING
}

\author{
TÍMEA HASZPRA*, PÉTER KISS ${ }^{\dagger}$, TAMÁS TÉL* \\ and IMRE M. JÁNOSI $I^{\dagger, \ddagger}$ \\ *Department of Theoretical Physics, \\ ${ }^{\dagger}$ Department of Physics of Complex Systems, \\ Eötvös Loránd University, Pázmány Péter sétány 1/A, \\ Budapest, H-1117, Hungary \\ ${ }_{j}^{\ddagger}$ janosi@lecso.elte.hu
}

Received March 1, 2011; Revised November 10, 2011

\begin{abstract}
Extensive numerical experiments are performed on tracer dispersion in global reanalysis wind fields. Particle trajectories are computed both along an isobaric $(500 \mathrm{hPa})$ and an isentropic $(315 \mathrm{~K})$ surface in a time interval of one year. Besides mean quantities such as advection of the center of mass and growth of tracer clouds, special attention is paid to the evaluation of particle pair separation dynamics. The characteristic behavior for intermediate time scales is Batchelor's dispersion along both surfaces, where the zonal extent of the tracer cloud increases linearly in time. The long-time evolution after 70-80 days exhibits a slower, diffusive dispersion (Taylor regime), in agreement with expectations. Richardson-Obukhov scaling (superdiffusion with an exponent of $3 / 2$ ) could not be identified in the numerical tests. The results confirm the classical prediction by Batchelor that the initial pair-separation determines subsequent time evolution of tracers. The quantitative dependence on the initial distance differs however from the prediction of the theory.
\end{abstract}

Keywords: Chaotic advection; geostrophic turbulence; pair dispersion; scaling.

\section{Introduction}

Tracer transport and mixing processes substantially influence the distribution of chemical compounds in geophysical flows, therefore the quantifying and modeling of related phenomena have a long history [Weiss \& Provenzale, 2008; Huang, 2010]. On the largest scales, the same governing equations are used both in the atmosphere and oceans, thus the tools to study tracer advection are also similar. Direct atmospheric experiments exist since the seventies, the first major balloon trajectory measurements were performed over the southern hemisphere: the EOLE at $200 \mathrm{mb}$ [Morel \& Bandeen, 1973; Morel \& Larcheveque, 1974], and the TWERLE at $150 \mathrm{mb}$ pressure level [Julian et al., 1977; Er-El \& Peskin, 1981]. Besides numerical simulations [Rood, 1987; Staniforth \& Côté, 1991], laboratory models [Jánosi et al., 2010] provide a deeper insight into the physical basis of the key processes.

Here we report the results of numerical experiments of tracer dispersion in realistic midtropospheric wind fields (see Fig. 1). The key quantities of interest are the mean drift of the "center of mass" of a cloud of tracers, and the spread of the cloud related to the average pair separation. The literature on pair dispersion both in 2D and $3 \mathrm{D}$ turbulent flows is pretty controversial, in spite of the intense research in the past decades (see [Salazar \& Collins, 2009; Bourgoin et al., 2006] and references therein). The main difficulty is that the dynamics obeys a series of crossover behavior from 


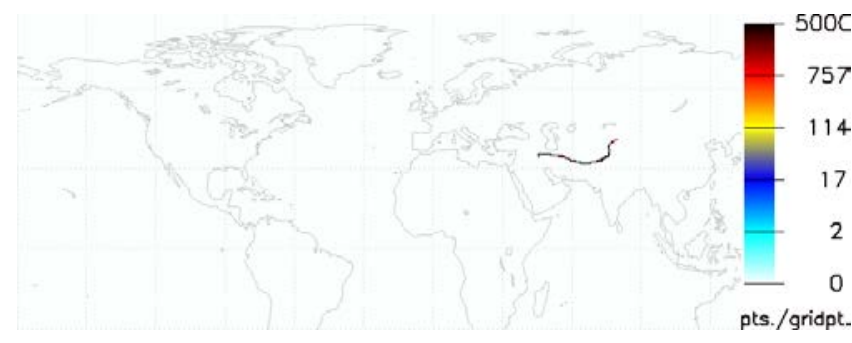

(a)

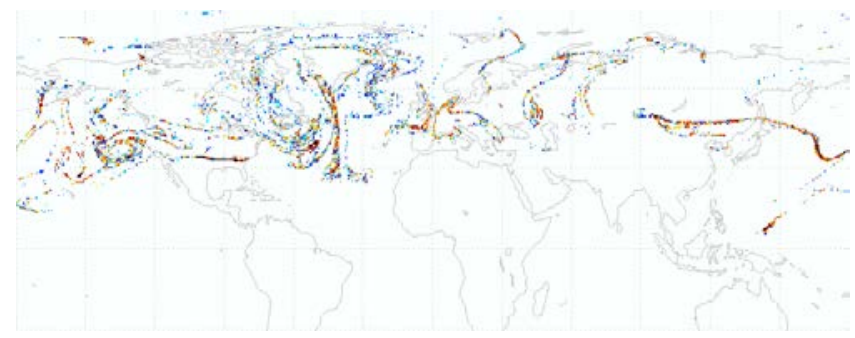

(c)

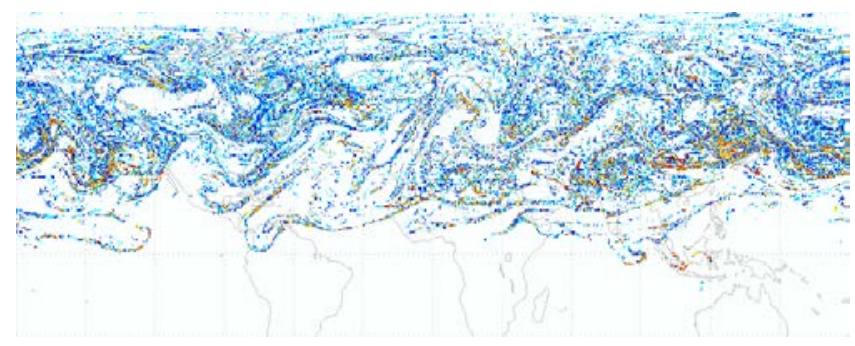

(e)

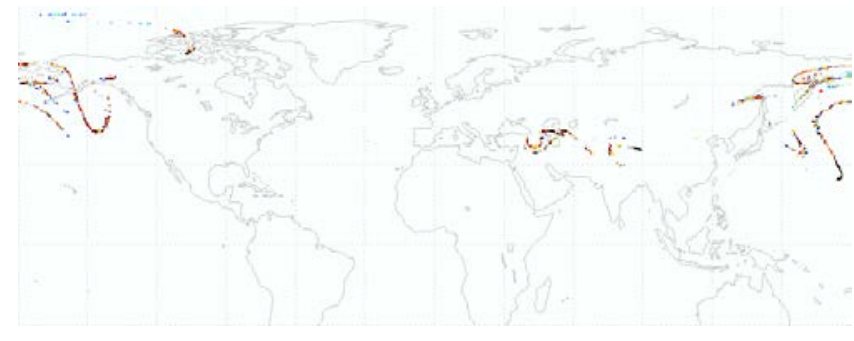

(b)

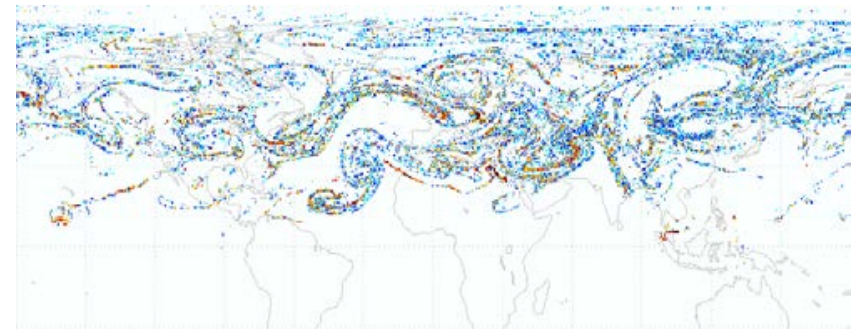

(d)

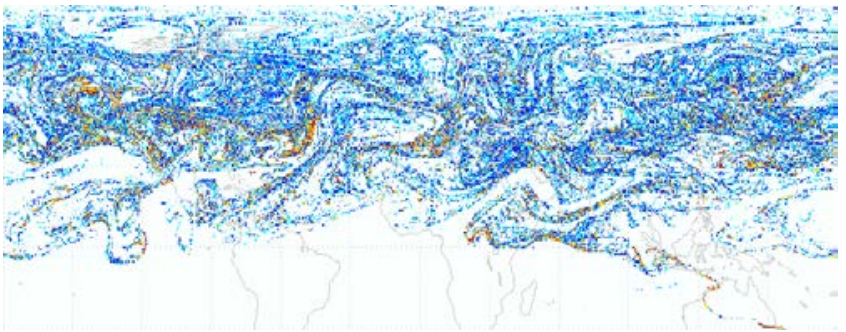

(f)

Fig. 1. Map sequence illustrating the dispersal of a small tracer cloud of $5 \times 10^{5}$ particles starting from $45^{\circ} \mathrm{N}, 0^{\circ}$ in a cell of size $0.5^{\circ} \times 0.5^{\circ}$ at $00 \mathrm{~h} \mathrm{UTC}, 01 / 01 / 2000,500 \mathrm{hPa}$ pressure level. Subsequent snapshots are separated by 7 days: (a) day 7 , (b) day $14, \ldots$ (f) day 42 . The color bar indicates tracer density measured as particle number in a cell of $0.5^{\circ} \times 0.5^{\circ}$.

exponential to power-law dispersion with various exponent values, and the crossover points cannot be easily located since they depend on several factors. Among others, the initial separation is an essential parameter, thus an extended tracer cloud exhibits almost always a mixed dynamics (nearby points behave differently from pairs of large initial separation). Recent challenging experiments have confirmed the classical theory by Batchelor [1950] that the time evolution strongly depends on the initial pair separation [Bourgoin et al., 2006].

The numerical experiments are based on the equations of passive scalar advection, where the background wind field is provided by the ERAInterim data bank of the European Centre for Medium-Range Weather Forecasts (http://www. ecmwf.int/research/era/). The advection along both isobaric and isentropic surfaces has almost the same dynamic features. The initial transients (short time dispersal) cannot be evaluated, because of the limited spatial and temporal resolution of the reanalysis wind field in the numerical tests. Pair separation is "ballistic" for intermediate time intervals, characterized by a linear growth of the zonal extent of the tracer cloud. This dynamics is not expected from theoretical considerations [Salazar \& Collins, 2009], but it is identified in other numerical [Huber et al., 2001; Haza et al., 2008] and experimental works [Bourgoin et al., 2006]. When strong correlations are produced by coherent structures in the flow decay at longer times, a gradual crossover to diffusive dispersal can be observed.

The paper is organized as follows. Section 2 gives an overview of the ECMWF reanalysis wind 
field and the numerical methods used in the trajectory calculations. The results are listed in Sec. 3, a detailed comparison with theoretical predictions is provided in Sec. 4. A summary is given in Sec. 5 .

\section{Data and Methods}

The accuracy of numerically computed tracer trajectories in various flow fields has improved a lot in the past decades [Stohl \& Seibert, 1998; Stohl, 1998; Weiss \& Provenzale, 2008]. In principle, precise atmospheric trajectories can be calculated directly from high resolution wind observations by interpolating between the measuring locations and time instants. In practice, however, trajectory calculations are mostly based on gridded output of numerical models (weather forecasts or reanalyses) such as provided by the European Centre for MediumRange Weather Forecasts (ECMWF) [Uppala et al., 2005].

In this work, the third generation ECMWF reanalysis ERA-Interim data bank is exploited, which is almost up to date from 01/01/1989 [http:// www.ecmwf.int/research/era/do/get/era-interim]. Zonal $(u)$ and meridional $(v)$ wind velocity components of global geographic coverage are evaluated for the years 2000 and 2009. Four values are available each day for $00 \mathrm{~h}, 06 \mathrm{~h}, 12 \mathrm{~h}$ and $18 \mathrm{~h}$ UTC (Universal Time Coordinated) at each geographic location with a spatial resolution of $1.5^{\circ} \times 1.5^{\circ}$ (lat/long). Note that the gridded wind fields are rather smooth, subgrid scale turbulence or vertical convection are not resolved. Although the wind velocity at a given site and time is intended to represent an instantaneous value [Uppala et al., 2005], direct comparison with high resolution wind tower measurements indicates that the velocity should be regarded as a six-hour mean value [Kiss et al., 2009].

Contrary to the horizontal wind velocity vector $(u, v)$, there are no routine observations for the vertical component $w$. Estimates can be produced by various meteorological models, but they are definitely less accurate than the fields of the horizontal wind. A plausible idea is to compute the 2D divergence field and estimate $w$ from the results, however it is known that this procedure has a very large error at the limited resolution of the reanalyses [Sardeshmukh \& Liebmann, 1993]. In the absence of reliable 3D wind fields, the usual procedure is to follow isobaric (constant pressure), or isentropic (constant potential temperature) surfaces with accommodating $2 \mathrm{D}$ wind velocity vectors. The latter has the advantage that atmospheric variables tend to be better correlated along isentropic surfaces than on constant pressure surfaces, however the potential temperature (entropy) of a given air parcel can significantly change when diabatic processes have an important role along a trajectory (e.g. in baroclinic stratification, which is common in mid-latitude tropospheric flows) [Stohl \& Seibert, 1998; Stohl, 1998]. For this reason we have computed and compared the statistical properties of tracer trajectories along both a constant pressure and a constant potential temperature level.

Global ERA-Interim wind fields are available on 37 pressure and 15 potential temperature levels in the ranges $1000-1 \mathrm{hPa}$, and $265-850 \mathrm{~K}$ [http:// data-portal.ecmwf.int/data/d/interim_daily/]. Since we are interested in free atmospheric flow, two levels in the mid-troposphere are selected for subsequent analysis. Firstly, the pressure level at $500 \mathrm{hPa}$ in the year 2000, and secondly, the isentropic level at $315 \mathrm{~K}$ in the year 2009. The former level is located in the range of 5-6 km altitude depending on latitude, season and weather. The $315 \mathrm{~K}$ potential temperature level usually intersects the $500 \mathrm{hPa}$ at around $30-45^{\circ}$ latitude on both hemisphere, it has lower values around the equator (down to $700 \mathrm{hPa}$ ), and higher values for high latitudes (up to $300-200 \mathrm{hPa}$ at the poles) (Fig. 2).

Trajectory calculation is based on the solution of the advection equation for a given infinitesimal air parcel [Ottino, 1989; Tél \& Gruiz, 2006]:

$$
\frac{d \mathbf{r}(t)}{d t}=\mathbf{v}(\mathbf{r}(t), t)
$$

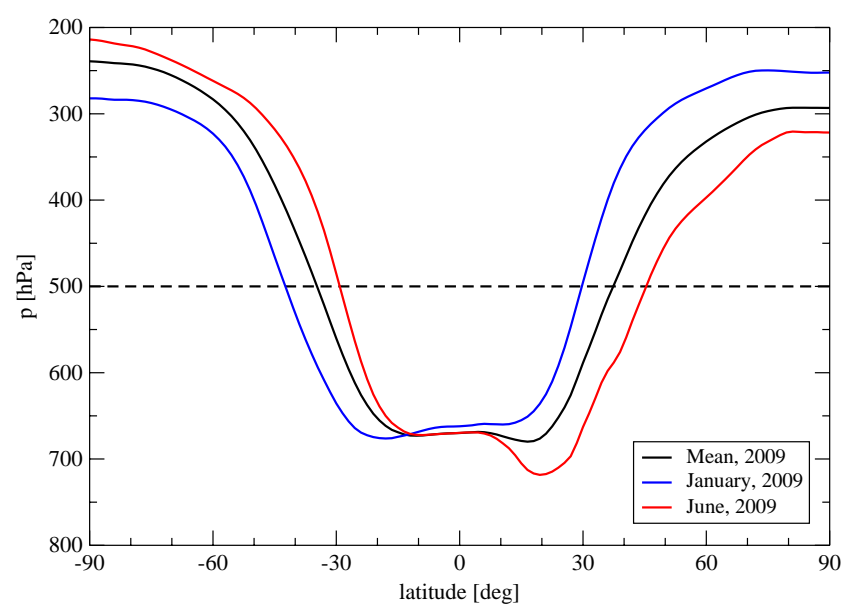

Fig. 2. Zonal mean pressure of the $315 \mathrm{~K}$ surface in the year 2009. The dashed line represents the isobaric surface used. 
where $\mathbf{v}(\mathbf{r}(t), t)$ is the instantaneous velocity field at the position $\mathbf{r}$ and time $t$ and $\mathbf{r}(t)$ is the trajectory. Equation (1) can be solved analytically for simple flow fields only, more realistic situations require a numerical treatment based on some finite difference Taylor-series representation [Stohl, 1998].

Our primary goal is to give a statistical characterization of atmospheric dispersal instead of calculating accurate trajectories (which is hopeless in view of the chaotic nature of the advection dynamics), therefore, we use the simplest approximations wherever possible. The temporal and spatial resolutions of ERA-Interim wind fields represent the most significant limitation, therefore very accurate atmospheric trajectories cannot be expected even by the most advanced numerical methods. It is also meaningless to compare trajectories starting from the same initial location but advancing on the two different surfaces, therefore the investigated temporal periods are also separated.

The limited spatial and temporal resolutions require the implementation of some interpolation procedures for the wind field at the numerical solution of Eq. (1). Several methods are known and tested in the literature [Rood, 1987; Staniforth \& Côté, 1991; Stohl et al., 1995]. For the time variable $t$, the simple linear interpolation provides a sufficiently accurate solution [Stohl et al., 1995]. Computationally more demanding algorithms are used for the spatial interpolation, such as the inverse quadratic $\left(1 / r^{2}\right.$ weighting) or cubic spline approximations [Press et al., 1992]. As for the numerical integration, the simple Euler method is implemented for the isobaric, and the fourth order Runge-Kutta algorithm for the isentropic trajectory computations [Press et al., 1992]. Consistency is checked with various time-steps of 6,15 and 30 min (see below).

Usually it is convenient to apply the regular latitude-longitude grid to characterize the advection in the atmosphere. But when cell sizes enter the statistics, it can be problematic, e.g. a cell of $1^{\circ} \times 1^{\circ}$ has a much smaller area near the poles than at the equator. In order to avoid such a high variability of the cell sizes in, e.g. tracer density statistics, an alternative tiling of almost equal-area tetragons is also used (Fig. 3), which is constructed in the following way. Two sides of the cells are constituted by latitudes of the same angle difference $\Delta \theta$, e.g. $\epsilon$, as in the case of the regular latitude-longitude grid. At the equator the other two sides of a cell are assigned by meridians with the angle difference $\Delta \phi=\epsilon$. Since the area of a cell of a sphere of unit radius defined by meridians $\theta_{1}, \theta_{2}$ and latitudes $\phi_{1}, \phi_{2}$ is $\left(\theta_{2}-\theta_{1}\right)\left(\sin \phi_{2}-\sin \phi_{1}\right)$, we can construct cells the area of which are equal to that of a cell near the equator. The angle between the meridians of a cell becomes larger at higher latitudes (Fig. 3). Since a band of a latitude has to be filled with integer

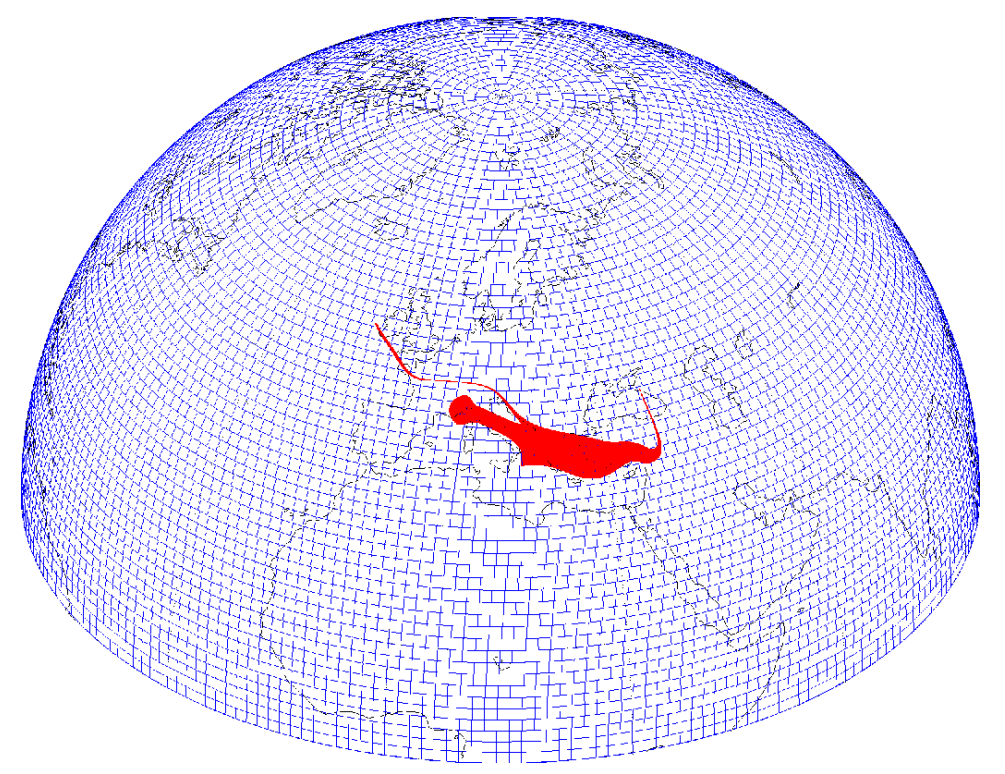

Fig. 3. Tiling of the Earth surface by almost equal-area tetragons. Red color marks an extended tracer cloud advected from $45^{\circ} \mathrm{N}, 0^{\circ}$ in a cell of size $10^{\circ} \times 10^{\circ}$ at 00 UTC $01 / 01 / 2009$ on $315 \mathrm{~K}$ level, after a day. 
number of cells, the cell sizes at different latitudes are not exactly the same.

\section{Results}

The time evolution of a small tracer cloud released from the middle of the northern hemisphere is illustrated in Fig. 1. Colors indicate the density computed as particle number in a given cell of $0.5^{\circ} \times 0.5^{\circ}$. Note that the meridional transport is strongly hindered across the equator, almost one year is necessary to obtain a near uniform tracer distribution on both hemispheres.

The following figures summarize the statistics of several numerical experiments. Note that the results are very similar for the isobaric and isentropic trajectories, thus we usually show plots for one case only, except when the definite goal is to illustrate close agreement. For the sake of simple identification, isobaric results are denoted by blue (turquoise error bars), while green indicates the results of isentropic calculations.

Figure 4 displays the time evolution of the center of mass $(\mathrm{CM})$ in the zonal and the meridional directions. An ensemble of 96 numerical experiments was evaluated. The center of 12 different clusters were located at equal longitudinal spacing of $30^{\circ}$ along the latitudinal circle $45^{\circ} \mathrm{N}$, and each initial configuration was simulated from eight different initial time instants for 20 days with a cloud of $10^{5}$ particles over an initial area of

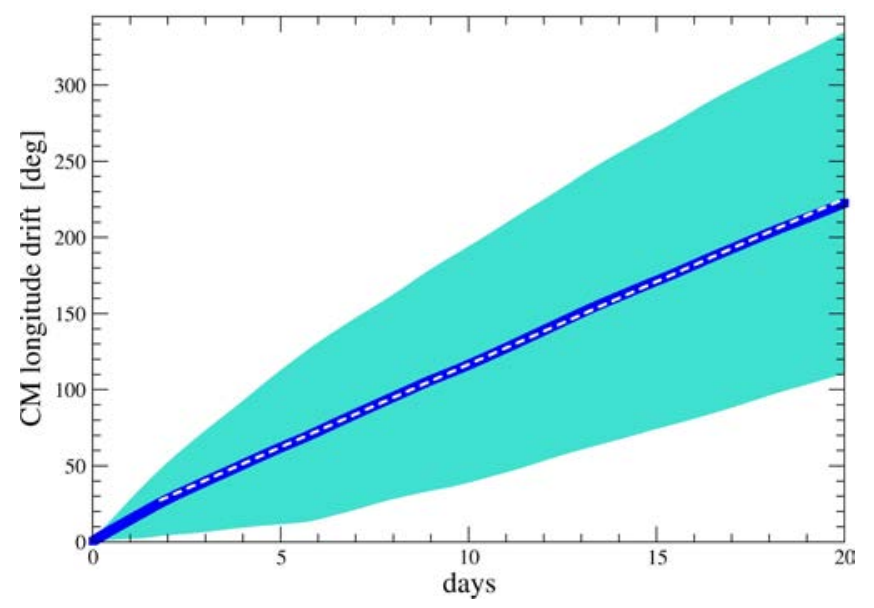

(a) $1^{\circ} \times 1^{\circ}$. The mean $\mathrm{CM}$ drift velocity at $500 \mathrm{hPa}$ has an almost constant eastward zonal component of $11^{\circ} /$ day $\left(\sim 870 \mathrm{~km} /\right.$ day at $\left.45^{\circ} \mathrm{N}\right)$ with a practically zero mean meridional component. The statistics is very similar over the $315 \mathrm{~K}$ isentropic surface, the mean zonal $\mathrm{CM}$ drift velocity is $14^{\circ} /$ day $\left(\sim 1107 \mathrm{~km} /\right.$ day at $\left.45^{\circ} \mathrm{N}\right)$ with zero meridional value. The larger value can be explained by the fact that the isentropic surface is situated at definitely higher altitudes over $45^{\circ} \mathrm{N}$, than the $500 \mathrm{hPa}$ isobar, thus a stronger eastward drift is expected.

This latter result is obtained from an ensemble averaging over 96 numerical experiments, where clusters of 130 particles distributed on a regular lattice inside of an initial cell of $10^{\circ} \times 10^{\circ}$ are studied with identical initial condition sets as above. The role of regular spacing was to avoid small tracer distances, we will return to this point in Sec. 4 .

The same ensemble was used to determine the zonal and meridional spreads of the tracer cloud. The edges of the clusters move with speeds different than that of the CM. The spreads $\theta(t)$ and $\phi(t)$ of the cloud is defined as the difference between the maximum and minimum angular coordinates of the cloud at a given time instant, in analogy with related laboratory experiments [Jánosi et al., 2010]. The average behavior is illustrated in Fig. 5: the zonal spreading quickly converges to a ballistic behavior of linear growth (slopes are $31.6^{\circ}$ /day for the isobaric, and $33.3^{\circ}$ day for the isentropic

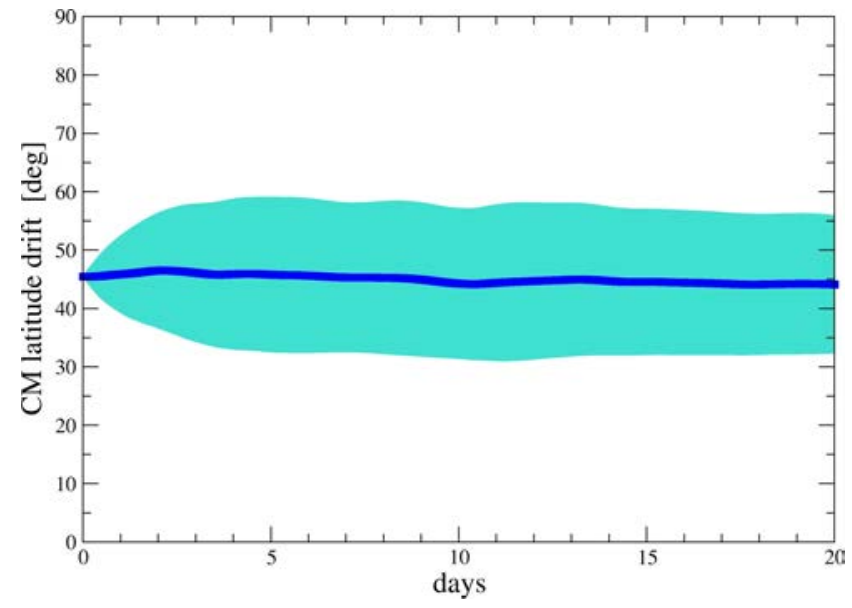

(b)

Fig. 4. Average time evolution of the center of mass (CM) for a tracer cloud formed by $10^{5}$ particles advected on the $500 \mathrm{hPa}$ pressure level. The initial area was $1^{\circ} \times 1^{\circ}, 12$ different initial longitude positions distributed evenly along the latitude $45^{\circ} \mathrm{N}$ and eight initial dates in the year 2000 formed the ensemble of 96 numerical experiments. (a) The mean zonal drift of CM (blue) and the standard deviation (turquoise). (b) The mean meridional drift of CM (blue) and the standard deviation (turquoise). 


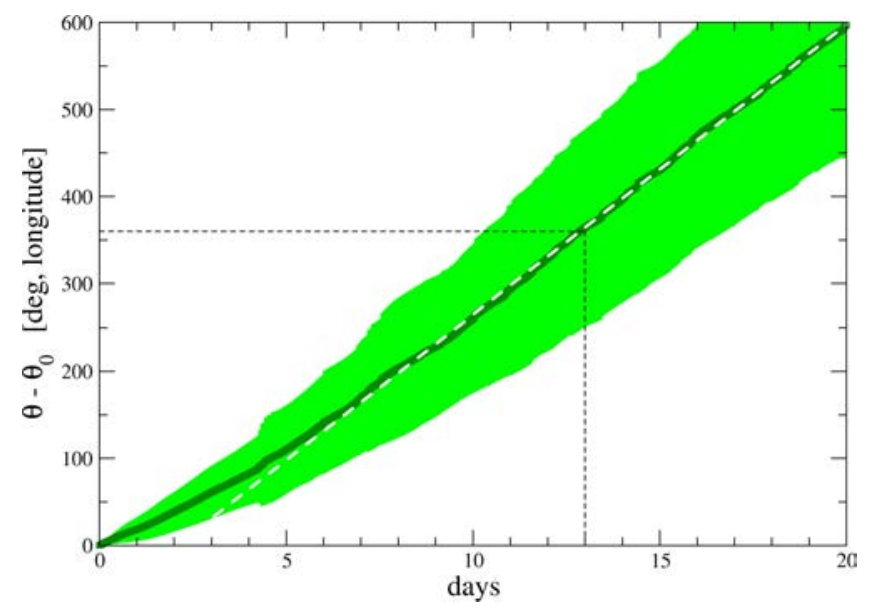

(a)

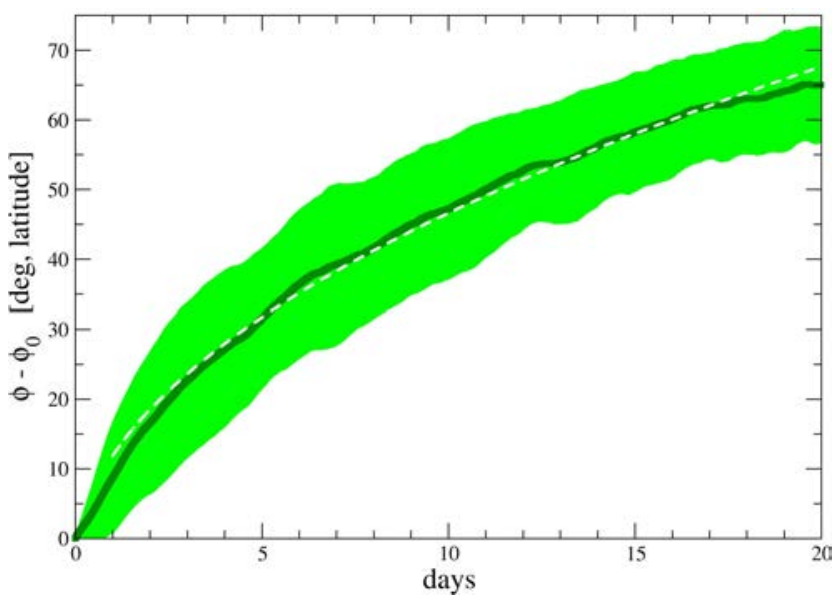

(b)

Fig. 5. Ensemble mean angular spread of the cloud in (a) zonal $\theta(t)$ and (b) meridional $\phi(t)$ directions. $\theta_{0}$ and $\phi_{0}$ mark the initial angular differences. Tracer clusters were formed by 130 particles, isentropic trajectories were evaluated. Light green band indicates one standard deviation obtained from 96 numerical experiments (see text). Black dashed line marks the average encompassing time of the globe $t^{*} \approx 13$ days in $(\mathrm{a})$, white dashed line is a linear fit in (a), and a square root fit in (b).

cases), while the meridional spread exhibits diffusive behavior with a $\sim \sqrt{t}$ growth after an initial transient of a couple of days.

It is interesting to compare the results of Fig. 5 with an alternative global measure of cloud size, the mean standard deviation of particle distances determined from the instantaneous center of mass. The results are plotted in Fig. 6. As in the case of the angular spread, the zonal mean standard deviation of particle distances increases linearly in time $\left(\approx 6^{\circ} /\right.$ day on the $315 \mathrm{~K}$ isentropic surface), while the meridional spread is $\sim \sqrt{t}$. This is in agreement

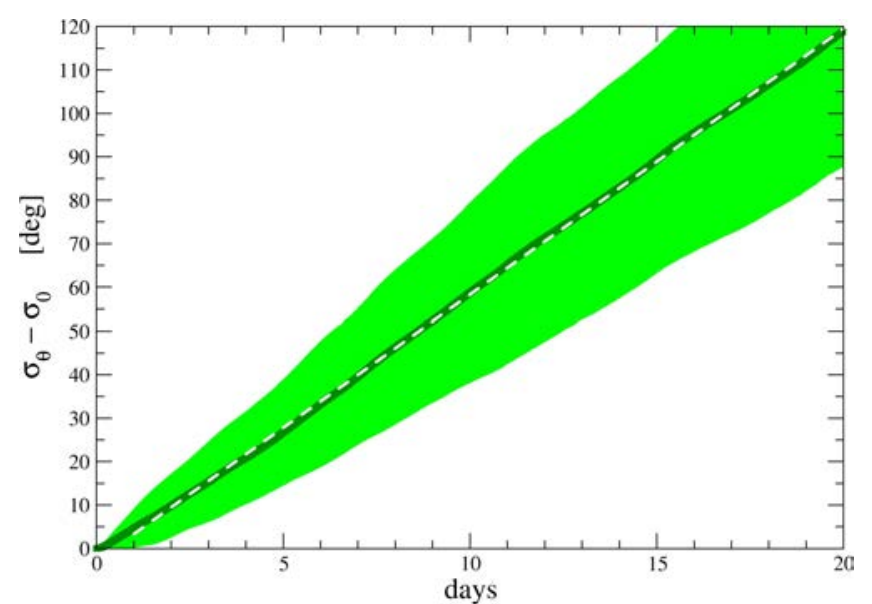

(a) with other studies [Pierrehumbert \& Yang, 1993; Huber et al., 2001].

Note that the measures of cloud extent defined by the edges or the standard deviation from the instantaneous center of mass provide a sufficient characterization for objects of compact shapes. However, tracer "clouds" in the atmosphere have a filamentary, fractal structure [Newell et al., 1992; Pierrehumbert \& Yang, 1993; Yang \& Pierrehumbert, 1994] (see Fig. 1), thus other measures might easily have different time evolutions. Such a measure, the covered area fraction $A$ is determined

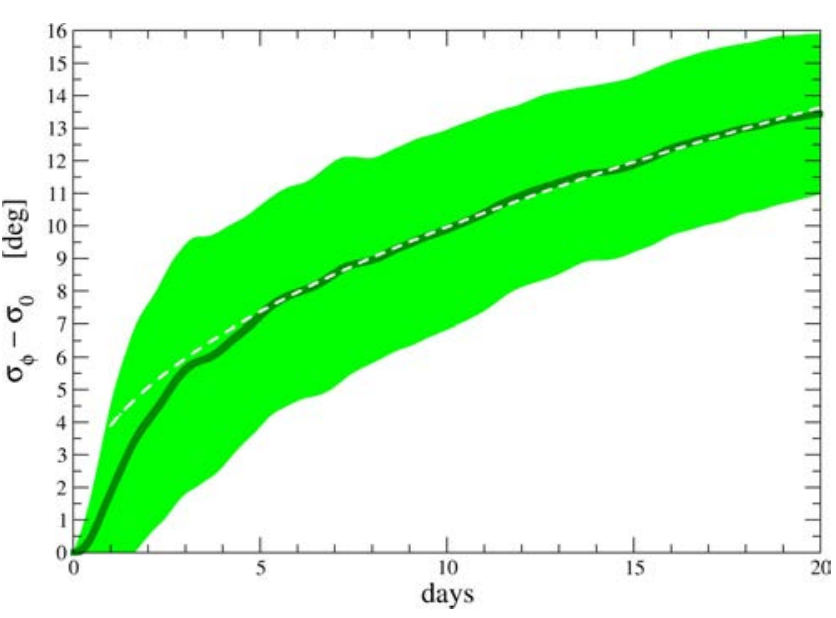

(b)

Fig. 6. Time evolution of the mean standard deviation of (a) zonal $\left(\sigma_{\theta}\right)$ and (b) meridional $\left(\sigma_{\phi}\right)$ distances measured from the center of mass. 96 isentropic experiments were evaluated in 2009 with 130 particles, and initial cloud size $10^{\circ} \times 10^{\circ}($ therefore the initial value is $\sigma_{0}=2.8767^{\circ}$ in both directions). White dashed line denotes (a) linear, and (b) square root fit from day five. 


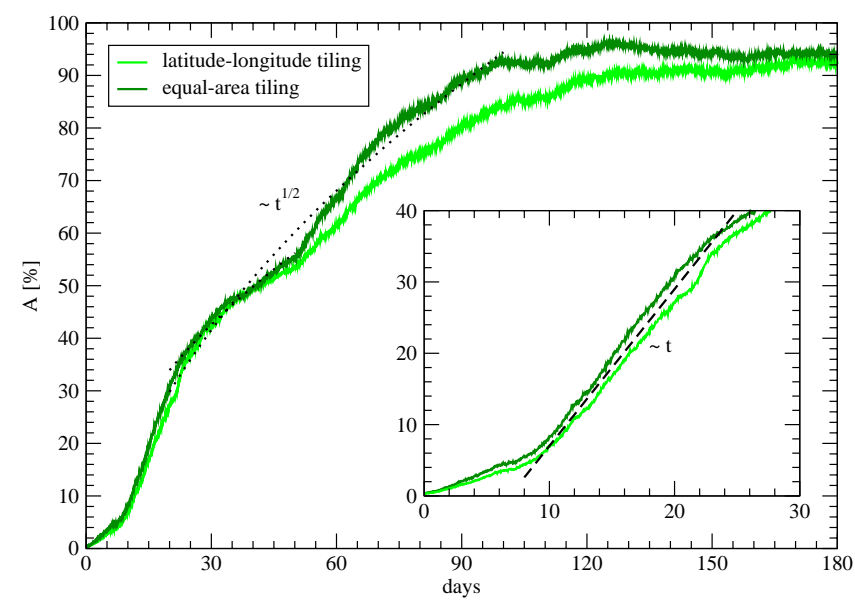

Fig. 7. Time evolution of the covered area fraction of a $10^{\circ} \times 10^{\circ}$ cloud started from $45^{\circ} \mathrm{N}, 0^{\circ}, 315 \mathrm{~K}, 01 / 01 / 2009$, $6.4 \times 10^{5}$ particles. Light green line represents the covered area fraction calculated from the regular latitude-longitude grid, while dark green line illustrates that from the equalarea tiling (3). Dotted lines illustrate asymptotic diffusive tendencies $(\sim \sqrt{t})$, the inset illustrates linear growth during the first month.

by the number of cells containing at least a single particle, normalized by the total cell-number of the global mesh. Similarly to the laboratory experiments [Jánosi et al., 2010], ballistic (linear) growth appears on an intermediate time interval of 10-20 days, see the inset in Fig. 7. The longtime growth after about two encompassing times ( $\sim 30$ days) is close to be diffusive, as expected.

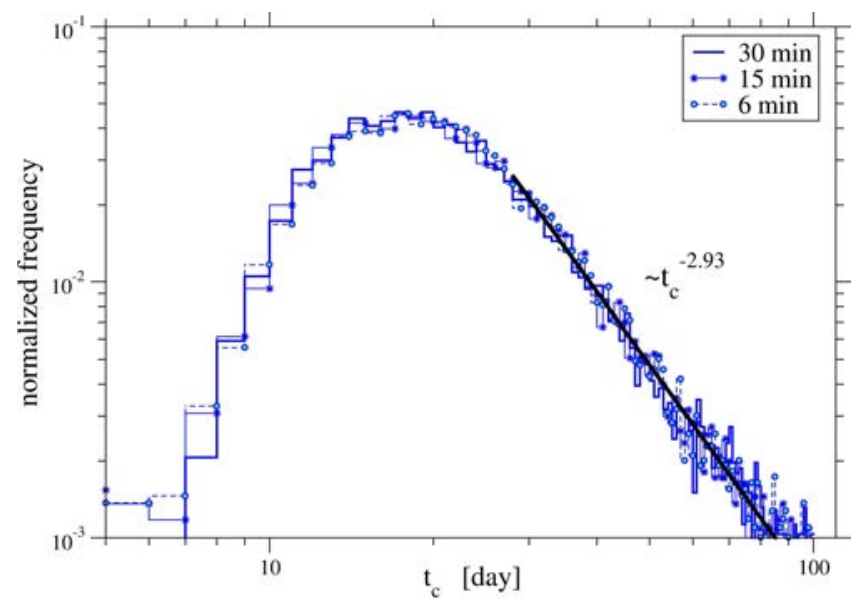

(a)
The numerical value of covered area fraction depends on the definition of the cells, but in the first two months and after 5-6 months (by the time the particles of the cloud have covered almost both hemispheres) the difference between the curves is small. At intermediate times the difference is due to the fact that the particles of the cloud reach the polar region, where the size of the cells is quite different in the two tilings. Naturally, in the regular latitude-longitude mesh the particles can fill more lower number cells near the pole, than in the equal-area tiling, when only few particles are advected there.

In Fig. 7 one can see that after about 2530 days the extent of the cloud increases slower than before. Then, after 50-60 days, it grows again approximately as fast as in the first month. The cause of this slowdown may be that after about a month the cloud covers almost the whole Northern Hemisphere $(A \approx 40-50 \%)$, but when the particles cross the equator and spread also in the Southern Hemisphere the spreading is rather slow due to the trade-wind belt. When they ultimately cross the barrier between the tropics and extratropics [Pierrehumbert \& Yang, 1993] also in the Southern Hemisphere, the covered area fraction increases again faster due to the stronger mixing effect of the midlatitude cyclones.

In Fig. 8, the circumnavigation (or zonal return) time $t_{c}$ for each individual tracer particle

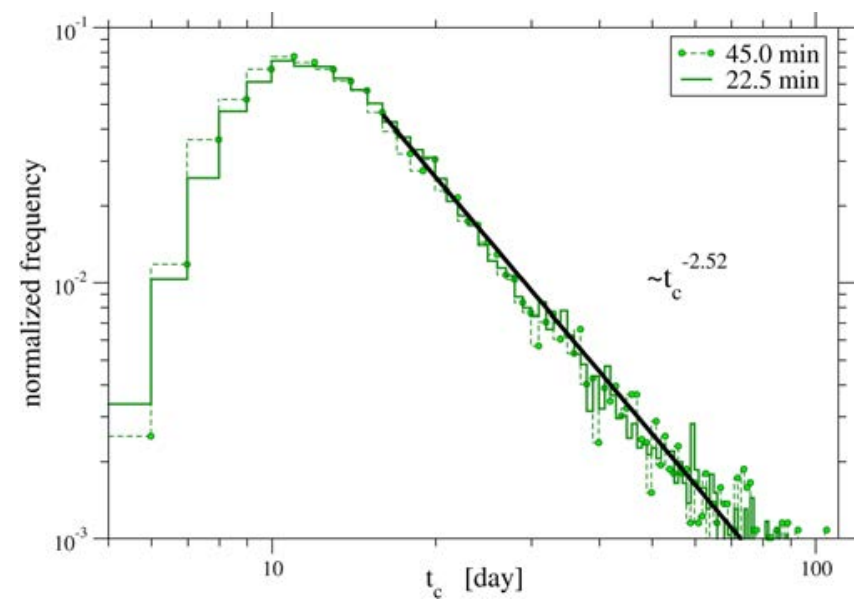

(b)

Fig. 8. (a) Empirical probability density distribution of the circumnavigation time $t_{c}$ for three numerical experiments at $500 \mathrm{hPa}$ with $10^{3}$ tracers and temporal resolutions of $6 \mathrm{~min}, 15 \mathrm{~min}$, and $30 \mathrm{~min}$ (Euler integration). The initial condition was a uniform spatial distribution along the longitude $0^{\circ}$ in the range of $\left[80^{\circ} \mathrm{S}, 80^{\circ} \mathrm{N}\right]$ latitude. (b) The same as (a) on the isentropic level of $315 \mathrm{~K}$ with two time steps $22.5 \mathrm{~min}$ and $45 \mathrm{~min}$ (fourth order Runge-Kutta integration). In both cases, the decay obeys power-law for large $t_{c}$ values (note the double-logarithmic scale). 


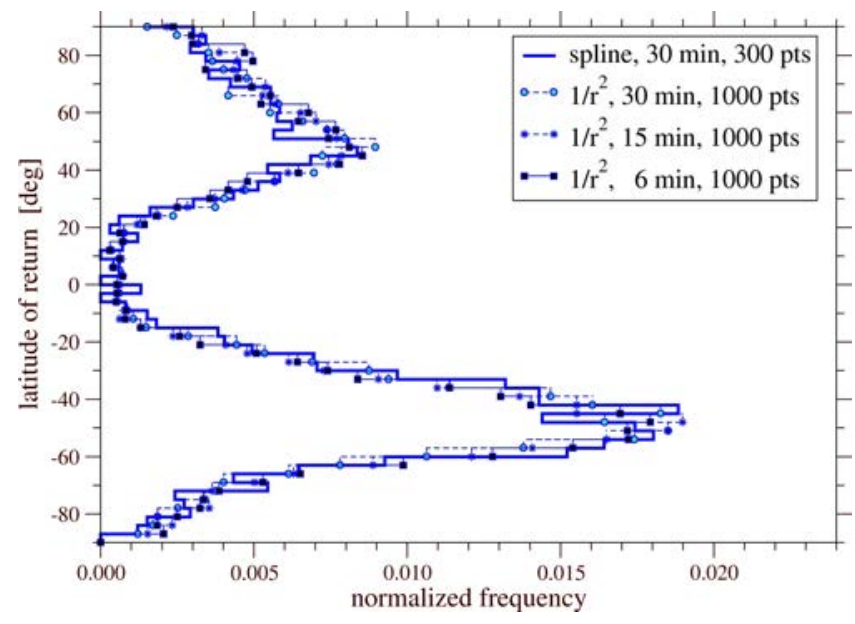

(a)

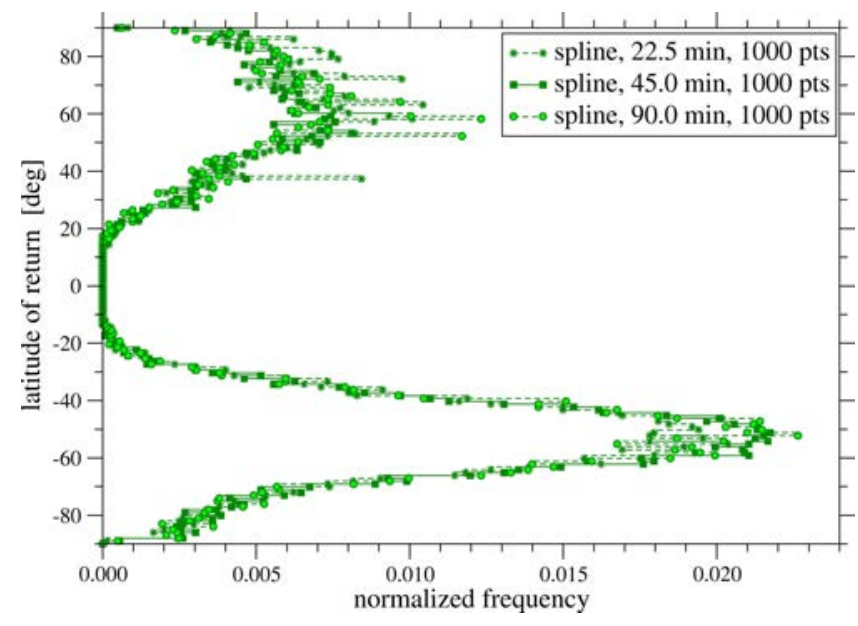

(b)

Fig. 9. Asymptotic empirical probability density distribution of the latitude of return on an inverted scale. Seven different numerical experiments are performed with different spatial interpolation schemes, integration time-steps, and cluster sizes (see legend). The initial condition was a uniform meridional distribution along the longitude $0^{\circ}$ in the range of $\left[80^{\circ} \mathrm{S}, 80^{\circ} \mathrm{N}\right]$ latitude. (a) $500 \mathrm{hPa}$, and (b) $315 \mathrm{~K}$.

is evaluated. The initial configuration was a material line of uniformly distributed tracers at $0^{\circ}$ (longitude) over the latitude range of $80^{\circ} \mathrm{S}-80^{\circ} \mathrm{N}$. The circumnavigation time $t_{c}$ has a very broad distribution, as illustrated in Fig. 8. Note that the encompassing time $t^{*}$ of a dye cloud is related to the width of the distribution of the single-particle circumnavigation time $t_{c}$, which reflects the difference between the fastest and slowest tracers in a given cloud. This quantity has a definite latitude dependence, as expected, because the meridional drift is a relatively slow diffusive process, and obviously a full circle takes a longer time near the equator than close to the poles. Note the consistency of the normalized histograms obtained at different time steps of the numerical integration. The tail of the empirical histograms obeys power-law decay with an exponent $\approx-2.9$ for the isobaric, and $\approx-2.5$ for the isentropic case. Due to the heavy tail, the mean value circumnavigation time $\left\langle t_{c}\right\rangle$ is larger than the mode (the most probable value) $\hat{t}_{c}$. It is remarkable that the mean value is almost the same $\left(\left\langle t_{c}\right\rangle \approx 23.5\right.$ days for isobaric, and $\left\langle t_{c}\right\rangle \approx 23.2$ days for isentropic trajectories), while the modes differ by almost a factor of two: $\hat{t}_{c} \approx 18.7$ days for isobaric, and $\hat{t}_{c} \approx 11.4$ days for isentropic runs.

Figure 9 illustrates an interesting focusing effect in the atmosphere: tracers that started from a uniform meridional distribution have a tendency to drift towards the mid-latitudes on both hemispheres during the advection. The results of seven experiments with different integration time-steps and spatial interpolation methods (inverse quadratic and cubic spline) are plotted together to demonstrate again statistical consistency. A cumulative distribution is obtained during one year of advection, by recording the latitude at each crossing of $0^{\circ}$ longitude. A similar focusing effect was reported in [Pierrehumbert \& Yang, 1993] by simulating trajectories over isentropic surfaces, however they studied tracer density distributions at fixed time differences.

\section{Batchelor's Scaling}

The motion of an incompressible turbulent fluid is described by the Navier-Stokes equation amended by the condition of zero divergence. According to Kolmogorov's (K41) similarity theory [Pope, 2000], the largest spatial and temporal scales are given by the energy-injection length scales $\mathcal{L}$ and eddy turnover time $T_{\mathcal{L}}$, respectively, while the smallest scales are the Kolmogorov length $\eta=\left(\nu^{3} / \epsilon\right)^{1 / 4}$ and the Kolmogorov time $\tau=\sqrt{\nu / \epsilon}$ (where $\nu$ is the kinematic viscosity, and $\epsilon$ is the constant energy dissipation rate per unit mass). The interval $(\eta, \mathcal{L})$ is known as the inertial subrange, because viscous dissipation becomes important only at length scales $l<\eta$.

Theoretical description of the mean square separation between two fluid elements $\left\langle r(t)^{2}\right\rangle$ in the inertial subrange is dated back to 1926, when 
Richardson suggested that it should grow in time as $t^{3}$ [Bourgoin et al., 2006; Ouellette et al., 2006]. Obukhov specified that in homogeneous and isotropic 3D turbulence, the pair dispersion should grow as $\left\langle r(t)^{2}\right\rangle=g \epsilon t^{3}$, where $g$ is a universal constant. Batchelor refined this work [Batchelor, 1950] by considering the role of initial separation $r_{0} \equiv r(t=0)$, and concluded that the mean square separation should grow as $t^{2}$ for times shorter than a characteristic timescale $t_{0}=\left(r_{0}^{2} / \epsilon\right)^{1 / 3}$ :

$$
\left[\left\langle r(t)^{2}\right\rangle-r_{0}^{2}\right]=\frac{11}{3} C_{2}\left(\epsilon r_{0}\right)^{2 / 3} t^{2}
$$

where $C_{2} \approx 2.13$ is the scaling constant for the second-order Eulerian velocity structure function [Bourgoin et al., 2006], and $t \ll t_{0}$. In the classical K41 theory of turbulence, $t_{0}$ may also be identified as the time for which the divergence of two fluid elements is determined by their initial relative velocity $\Delta \mathbf{v}_{0}$ in a given eddy of size $r_{0}$. For times $t>t_{0}$, the growth of the pair separation is expected to follow the Richardson-Obukhov scaling, independently of $r_{0}$. When the separation exceeds the size of largest coherent structures, diffusive dispersion is expected $\left\langle r(t)^{2}\right\rangle \sim t$ (Taylor regime). In statistically stationary forced homogeneous and isotropic twodimensional turbulence, the Richardson-Obukhov $t^{3}$ scaling holds, however experiments found various empirical exponent values between 2 and 3 [Salazar \& Collins, 2009; Tabeling, 2002].

An essential condition behind the validity of the Batchelor scaling Eq. (2) is the lack of correlations between the initial separation $\mathbf{r}_{0}$ and the relative velocity of the pair $\Delta \mathbf{v}_{0}$ [Ouellette et al., 2006]. When this condition is not fulfilled, the correct scaling form of relative pair separation is based on the vectorial difference as

$$
\left\langle\left|\mathbf{r}(t)-\mathbf{r}_{0}\right|^{2}\right\rangle=\frac{11}{3} C_{2}\left(\epsilon r_{0}\right)^{2 / 3} t^{2} .
$$

Note that these Batchelor forms bear important consequences related to the evaluation of experimental data. (i) For fixed energy dissipation rate per unit mass $\epsilon$, the rate of pair separation depends on the initial value $r_{0}$ itself. (ii) The transition time to the Richardson-Obukhov scaling $t_{0}$ depends also on $r_{0}$. This means that one cannot observe a "clean" dynamics in a cloud of tracers with a mixture of various initial separations. (iii) Identification of the Richardson-Obukhov $t^{3}$ scaling requires also a significant time scale separation between $t_{0}$ and the eddy turnover time $T_{\mathcal{L}}$ [Ouellette et al., 2006]. Since the latter quantity is estimated about $3-5$ days in the troposphere (considering scales of a typical midlatitude cyclone) [Gerber \& Vallis, 2007], and values for $\epsilon$ are around $10^{-5} \mathrm{~m}^{2} / \mathrm{s}^{3}$ [Lilly, 1983], an initial pair separation of $r_{0} \approx 300-500 \mathrm{~km}$ easily produces $t_{0}$ more than a day.

Here we show representative results for $96-96$ isobaric and isentropic dispersal simulations, where the average pair separation is determined by both the scalar and the vectorial definitions Eqs. (2) and (3). Individual clusters (12 locations and 8 starting time instants, as before) are formed by 130 regularly spaced tracers over a geographic area of $10^{\circ} \times 10^{\circ}$. The time dependence of pair separation was determined in initial distance bins of $50 \mathrm{~km}$, where the central value of the bins are indicated in the legend of Figs. 10(a), 10(c) and 10(e) (thus bin "75 km" denotes $50 \mathrm{~km}<r_{0}<100 \mathrm{~km}$, etc., except the largest value where " $975 \mathrm{~km}$ " means $\left.r_{0}>950 \mathrm{~km}\right)$.

A special feature emerges for the vectorial difference in Eq. (3), because pair dispersion occurs over a spherical surface. The definition for $\left[\mathbf{r}(t)-\mathbf{r}_{0}\right]$ is unambiguous in 3D Euclidean sense, however pair distances must be determined along geodesics in the atmosphere at large enough separations. To avoid computational discrepancies, we adopted the following convention. One of the points of each pair is selected, and the angles are calculated in the comoving Euclidean frame of reference (zonal $x$ and meridional $y$ axes) with the origin at this point. The lengths of vectors are calculated along geodesics. We checked that the statistics conforms with Eq. (2) where scalar separations were computed by pure spherical geometry.

The results in Figs. 10(a), 10(c) and 10(e) are consistent with the Batchelor hypothesis over almost two decades of the time axis: the mean squared separation increases with $t^{2}$, and the slopes depend on the initial value $r_{0}$. We emphasize that the behavior is almost the same for both the scalar and vectorial distances [Eqs. (2) and (3)], apart from numerical values for the slopes shown in Fig. 10(f). The empirical scaling with $r_{0}$ is somewhat surprising, because the found exponent $\sim 5 / 3$ is very far from the $2 / 3$ of the theory [see Eqs. (2) and (3)], and this cannot be a simple consequence of numerical inaccuracies or statistical errors. We are fully aware of the fact that large scale dispersal in the atmosphere (and in the laboratory tank) can 

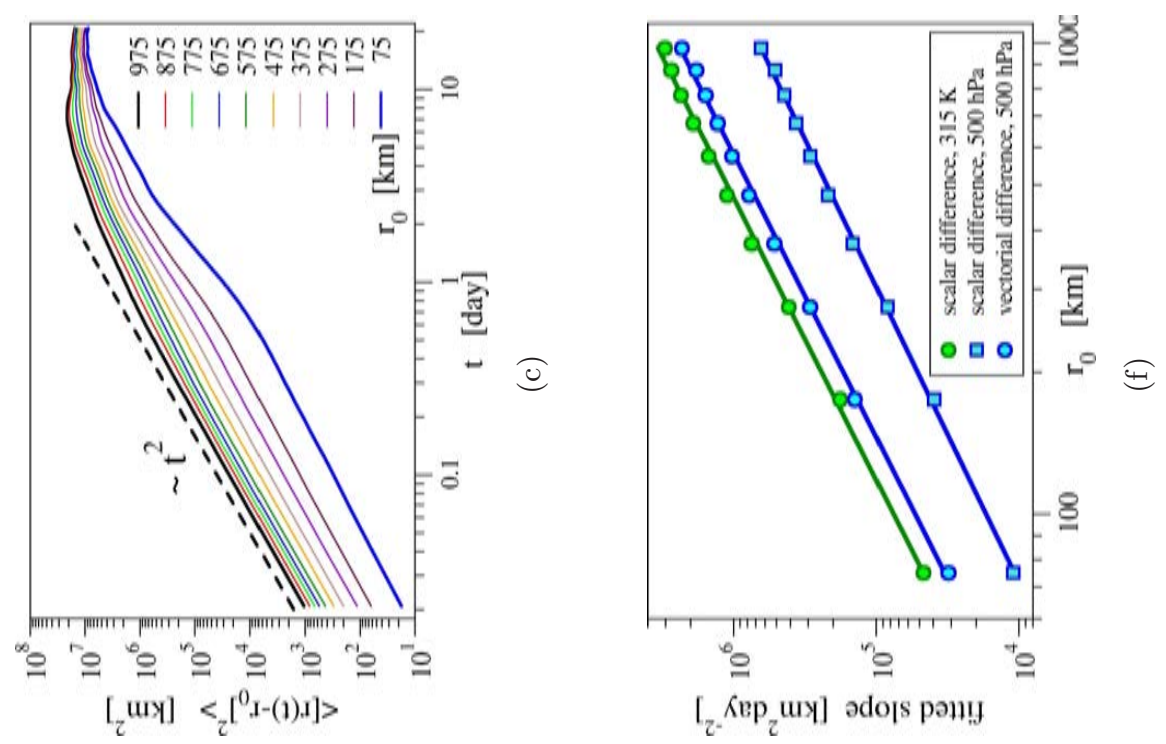

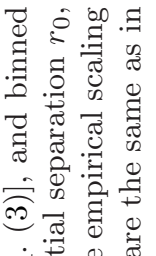

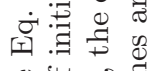

\&

.

릴

穵 क ठै है है

ज्ञ.

ن.

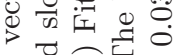

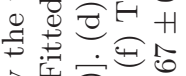

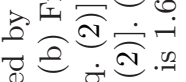
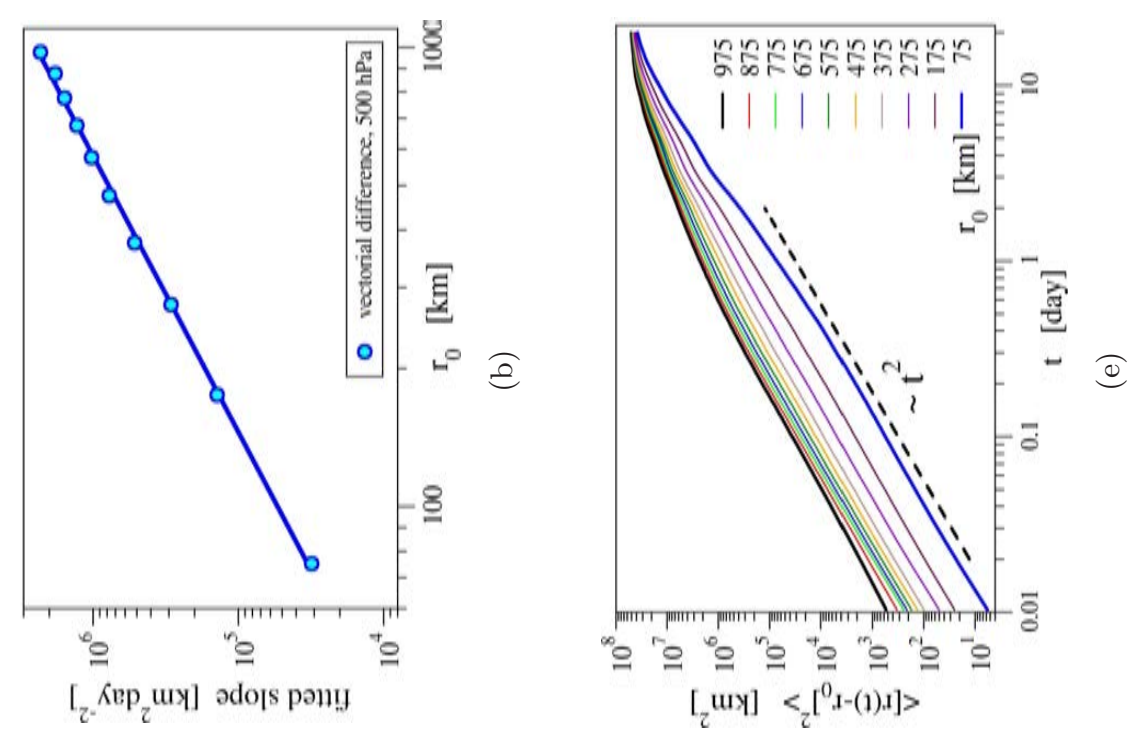

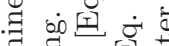

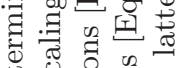

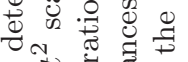

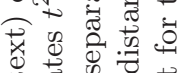

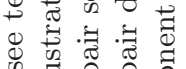

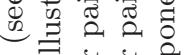

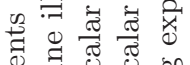

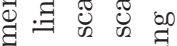

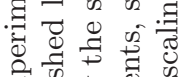

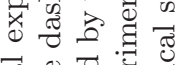

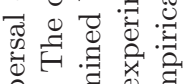

के है वै

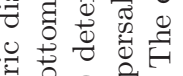

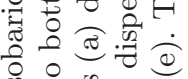

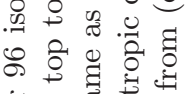

点

영
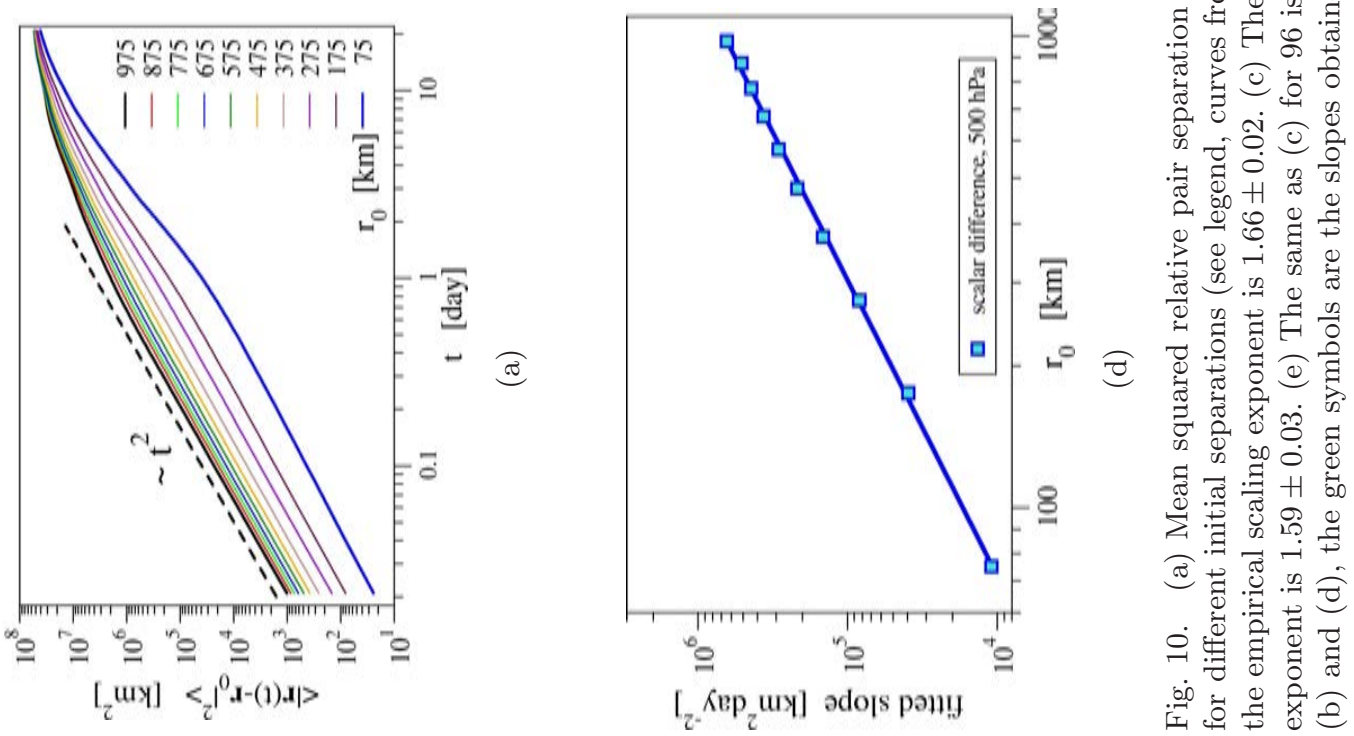

[z- $\mathrm{Rep}$ uy] әdo[s pəu!

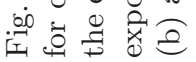


be different from what one expects in 3D homogeneous and isotropic turbulence, still the theoretical predictions concerning the time dependence are very similar in both cases.

\section{Summary}

Here we summarize our findings for the Batchelor scaling in a compact form.

- Important results of the numerical advection tests are that the mean pair separation strongly depends on the initial value $r_{0}$ and follows a ballistic growth [Figs. 10(a), 10(c) and 10(e)], similarly to the Batchelor hypothesis. A relevant consequence is that an extended tracer cloud containing different values of $r_{0}$ exhibits always a mixed behavior, therefore a direct relationship between pair separation and overall statistics cannot be easily formulated.

- The slopes of the mean pair separation squares versus $t^{2}$ lines do not follow the Batchelor scaling, the apparent exponent value is $\approx 5 / 3$ [Fig. 10(f)]. In contrast to Eqs. (2) and (3), simple dimensional considerations with this exponent suggest a combination like $U^{-3}\left(\epsilon r_{0}\right)^{5 / 3}$, where $U$ has a dimension of velocity. However, the appearance of such a factor seems a challenge. Further work is needed to explain these observations.

- The quasigeostrophic turbulent wind field is far from being homogeneous and isotropic, large scale (irregular) eddies determine the dynamics. In addition, reanalysis fields do not resolve structures below the grid spacing. Consequently, the collective dynamics of an extended tracer cloud is spoiled by pairs of initial separation below gridsize. An obvious sign of this effect is the appearance of unrealistically long initial transients at too small cloud sizes, where the "true" dynamics unfolds only when the mean pair separation definitely exceeds the cell size as also reported in [Jánosi et al., 2010]. This observation might help to clarify earlier results on scaling behavior of tracers simulated in wind fields of limited resolutions [Huber et al., 2001; Pierrehumbert \& Yang, 1993].

- In Sec. 3 of the 1950 key paper by Batchelor (The applicability of the similarity hypotheses to the turbulence in the atmosphere and ocean), a very conservative estimate for the atmospheric inertial subrange spans between $0.002-100 \mathrm{~m}$. Indeed, this is far from the scales we used in our simulations, where the lower limit is determined by the spatial resolution of the global wind field, and the upper limit is given by the estimate of typical eddy turnover time by Gerber and Vallis [2007]. Note however, that homogeneity and isotropy are statistical terms, and any given instantaneous snapshot of a turbulent field exhibits coherent structures on an extremely wide range. For the very reason, scaling laws can be formulated only for mean quantities (individual pair separation trajectories have fluctuation in a wide range clearly seen in Figs. 4-6). Our observation of the Batchelor type scaling is purely empirical, we did not use any additional assumption or filtering, apart from taking care of the initial pair separation. It is highly possible that the anomalous exponent value is an inherent property of quasigeostrophic turbulence, however we cannot give an easy explanation.

\section{Acknowledgments}

This work was supported by the Hungarian Science Foundation (OTKA) under Grant Nos. NK72037 and NK100296, by the European Commission's COST Action MP0806, and by the European Union and the European Social Fund under the grant agreement no. TÁMOP 4.2.1./B-09/KMR-20100003.

\section{References}

Batchelor, G. K. [1950] "The application of the similarity theory of turbulence to atmospheric diffusion," $Q . J$. Roy. Meteorol. Soc. 76, 133-146.

Bourgoin, M., Ouellette, N. T., Xu, H., Berg, J. \& Bodenschatz, E. [2006] "The role of pair dispersion in turbulent flow," Science 311, 835-838.

Er-El, J. \& Peskin, R. L. [1981] "Relative diffusion of constant-level balloons in the Southern hemisphere," J. Atmos. Sci. 38, 2264-2274.

Gerber, E. P. \& Vallis, G. K. [2007] "Eddy zonal flow interactions and the persistence of the zonal index," J. Atmos. Sci. 64, 3296-3311.

Haza, A. C., Poje, A. C., Özgökmen, T. M. \& Martin, P. [2008] "Relative dispersion from a high-resolution coastal model of the Adriatic Sea," Ocean Model. 22, $48-65$.

Huang, R. X. [2010] Ocean Circulation: Wind-Driven and Thermohaline Processes (Cambridge University Press, Cambridge, UK).

Huber, M., McWilliams, J. C. \& Ghil, M. [2001] "A climatology of turbulent dispersion in the troposphere," J. Atmos. Sci. 58, 2377-2394. 
Jánosi, I. M., Kiss, P., Homonnai, V., PattantyúsÁbrahám, M., Gyüre, B. \& Tél, T. [2010] "Dynamics of passive tracers in the atmosphere: Laboratory experiments and numerical tests with reanalysis wind fields," Phys. Rev. E 82, 046308.

Julian, P., Massman, W. \& Levanon, N. [1977] "The TWERL experiment," Bull. Am. Met. Soc. 58, 936948.

Kiss, P., Varga, L. \& Jánosi, I. M. [2009] "Comparison of wind power estimates from the ECMWF reanalyses with direct turbine measurements," J. Renew. Sust. Ener. 1, 033105.

Lilly, D. K. [1983] "Stratified turbulence and the mesoscale variability of the atmosphere," J. Atmos. Sci. 40, 749-761.

Morel, P. \& Bandeen, W. [1973] "The EOLE experiment: Early results and current objectives," Bull. Am. Met. Soc. 54, 298-306.

Morel, P. \& Larcheveque, M. [1974] "Relative dispersion of constant-level balloons in the $200 \mathrm{mb}$ general circulation," J. Atmos. Sci. 31, 2189-2196.

Newell, R. E., Newell, N. E., Zhu, Y. \& Scott, C. [1992] "Tropospheric rivers? A pilot study," Geophys. Res. Lett. 12, 2401-2404.

Ollitrault, M., Gabillet, C. \& Colin de Verdiere, A. [2005] "Open ocean regimes of relative dispersion," J. Fluid Mech. 533, 381-407.

Ottino, J. M. [1989] The Kinematics of Mixing: Stretching, Chaos, and Transport (Cambridge University Press, Cambridge, UK).

Ouellette, N. T., Xu, H., Bourgoin, M. \& Bodenschatz, E. [2006] "An experimental study of turbulent relative dispersion models," New J. Phys. 8, 109.

Pierrehumbert, R. T. \& Yang, H. [1993] "Global chaotic mixing on isentropic surfaces," J. Atm. Sci. 50, 24622480.

Pope, S. B. [2000] Turbulent Flows (Cambridge University Press, Cambridge, UK).

Press, W. H., Teukolsky, A. A., Vetterling, W. T. \& Flannery, B. P. [1992] Numerical Recipes in $C$ : The Art of
Scientific Computing, 2nd edition (Cambridge University Press, Cambridge, UK).

Rood, R. B. [1987] "Numerical advection algorithms and their role in atmospheric transport and chemistry models," Rev. Geophys. 25, 71-100.

Salazar, J. P. L. C. \& Collins, L. R. [2009] "Two-particle dispersion in isotropic turbulent flows," Ann. Rev. Fluid Mech. 41, 405-432.

Sardeshmukh, R. D. \& Liebmann, B. [1993] "An assessment of low-frequency variability in the tropics as indicated by some proxies of tropical convection," J. Climate 6, 569-576.

Staniforth, A. \& Côté, J. [1991] "Semi-Lagrangian integration schemes for atmospheric models - A review," Mon. Wea. Rev. 119, 2206-2223.

Stohl, A., Wotawa, G., Seibert, P. \& Kromp-Kolb, H. [1995] "Interpolation errors in wind fields as a function of spatial and temporal resolution and their impact on different types of kinematic trajectories," J. Appl. Meteorol. 34, 2149-2165.

Stohl, A. [1998] "Computation, accuracy and applications of trajectories - A review and bibliography," Atmos. Environ. 32, 947-966.

Stohl, A. \& Seibert, P. [1998] "Accuracy of trajectories as determined from the conservation of meteorological tracers," Q. J. Roy. Metorol. Soc. 124, 1465-1484.

Tabeling, P. [2002] "Two-dimensional turbulence: A physicist approach," Phys. Rep. 362, 1-62.

Tél, T. \& Gruiz, M. [2006] Chaotic Dynamics: An Introduction Based on Classical Mechanics (Cambridge University Press, Cambridge, UK).

Uppala, S. M. et al. [2005] "The ERA-40 re-analysis," Q. J. Roy. Meteorol. Soc. 131, 2961-3012.

Weiss, J. B. \& Provenzale, A. (eds.) [2008] Transport and Mixing in Geophysical Flows (Springer, Berlin, Germany).

Yang, H. \& Pierrehumbert, R. T. [1994] "Production of dry air by isentropic mixing," J. Atmos. Sci. 51, $3437-3454$. 